\title{
Model of Determination of the Dynamical Stability of Economic Systems
}

\author{
Elena Kostenko ${ }^{1, *}$, Vitaly Kostenko ${ }^{2}$, Volodymyr Kuznichenko ${ }^{3} \&$ Volodymyr Lapshyn ${ }^{3}$ \\ ${ }^{1}$ Manifest Communications Inc., 197 Spadina Avenue, Suite 500 Toronto, Ontario, Canada \\ ${ }^{2}$ Department of Electronics and Control Systems, V.N. Karazin Kharkiv National University, \\ 4 Svobody Sq., Kharkiv 61022, Ukraine \\ ${ }^{3}$ Department of Mathematical Methods in Economics and Information Technologies, Kharkiv \\ Institute of Finances USUFIT, 5 Pletnivs'ky Lane, Kharkiv 61003, Ukraine
}

*Corresponding author: Manifest Communications Inc., 197 Spadina Avenue, Suite 500Toronto, Ontario M5T 2C8, Canada. Tel: 1-416-593-7017 ext. 242 E-mail: elena_kostenko@manifestcom.com

Received: February 4, 2015 Accepted: April 16, 2015 Published: May 15, 2015

doi:10.5296/rae.v7i2.7048 URL: http://dx.doi.org/10.5296/rae.v7i2.7048

\begin{abstract}
The article proposes an algorithm to qualitatively determine the dynamic state of an economic system. The base data for the algorithm are the current values of the parameter that describes the deviation of the system from equilibrium. The method of phase planes is partially used. The interpolation of the statistical data of the system parameter as a function of time, as well as the parametric definition of the forces that act on the system, allows for the determination of its potential functions and points of equilibrium. The characteristics of the points of equilibrium, namely their extrema type, determine the areas of the systems stability and instability. The use of standard computer programs allows for the analytical and graphical (by analysing the form of the potential function) determination of the dynamic stability of a system, by looking at the current parameters of the system. A demonstration of the algorithm is given in an example.
\end{abstract}

Keywords: algorithm, dynamic state, economic system, equilibrium, stability. 


\section{Introduction}

The constantly changing internal and external parameters of economic systems lead to cyclical changes in their state. By studying the parameters of different cycles, we are able to (to a certain extent) predict the booms and busts of economies both global and local. This in turn gives the possibility of mitigating the negative effects that busts have on economies. The presentation of this is the goal of the present article.

After the works of Kondratief N., who discovered long-period cycles of development of the world economy, other periodic changes of macroeconomic and microeconomic systems were discovered, with smaller periods. In order to describe and predict these processes, different models are used.

Smirnov A. analysed macroeconomic dynamics based on non-linear differential equations. He also analysed already existing models of linear and non-linear dynamics of economic systems. These models, as well as the methods of their analysis in the presence of non-linearity and stochasticity, were proposed and were analysed by Goodwin R., Lorenz H-W, Verhulst F., Turnovsky S. The model of business activity was developed by Milovanov $\mathrm{V}$. He analysed questions related to market dynamics and price formation. The system of equations that described the changes in material and financial resources included non-linearity. As a result of the research of Forrester G. and Medouza D., a "dynamic model of global human development" was created. Peters A. analysed processes that occur on the financial market, economic cycles and crises based on non-linear economic dynamics. In the book of Punzo F., a collection of specialists' essays (Amendola M., Aoki M., Baumol W., Böhm B., Day R., Delli Gatti D., Flaschel P., Gaffard J.-Luc, Gallegati M., Hahn F., Hogg T., Huberman B., Iwai K., Pavlov O., Punzo L., Rissanen J.) contributes to an assessment of the understanding of the relationship between cycles, growth and structural change. The variety of approaches in this book represents the economists' views of the discussed area well: from neoclassical and endogenous growth theories, to classical, evolutionary and neo-Austrian dynamics; from real business cycle and information-based econometrics to other non-linear techniques related to complex dynamics.

The oscillations of economic systems around equilibrium can be both stable and unstable. Instabilities are, usually, related to non-linearities in the equations that describe the dynamics of the system. They can lead to unexpected changes in the state of these systems. This is the source of the enduring interest towards the analysis of equilibrium states of economic systems, their oscillations, and the internal and external parameters that cause them.

The business cycle associated with exchange rate-based inflation stabilization policy was analyzed by Rhee $\mathrm{H}$. The stochastic dynamic model of general equilibrium was developed. The effect of macroeconomic instability on economic growth was investigated by Haghighi H. Changes in macroeconomic instability indicators are associated with the increase (decrease) of economic growth in the long run. Combination of the commodity market and financial market equilibriums was investigated by Baba I.. The Investment Saving-Liquidity Preference Money Supply (IS-LM) model was used. This model is a macroeconomic tool that demonstrates the relationship between interest rates and real output in the commodity and services market and the financial market. The results of Mclean R. suggest that both the 
business cycle and investor sentiment have significant and independent effects on the cost of external finance. Results of Wan-Shin Mo. show that economic growth rates in both Hong Kong and Taiwan are well described by a threshold model but with different types of nonlinear effects.

Ukrainian scientists also contribute to the study of cyclical oscillations of economic systems and their parameters. Cyclical oscillations of the GDP, of the export and import, or consumer and investor spending, of the efficiency of employment and real salaries and of the index of consumer prices in Ukraine were analyzed by Pirtch M. The employment - one of the most important components of the economic system - is studied by Dankiv V. It is related to the cyclical oscillations of the market's present state, which has an effect on the dynamics of unemployment. A modified model of capital dynamics, which takes into account distributed delays during realization of investments, is presented by Vnukova N., wherein an analysis of observed cyclical changes in the accumulation of capital and investments is performed.

Different economic models (classical, Keynesian, Cobweb, monetary and others (Macconel C., Brownlee O., Brunner K., Tobin J., Ezekiel M., Ruth M., Friedman M.)) propose natural oscillations of the corresponding parameters of the employment, commodities and finance markets around their respective equilibrium points. They describe the dynamics qualitatively, not using a mathematical apparatus.

Therefore the cited sources, the list of which can be significantly expanded, confirm the importance of the methods of study of cyclical changes of parameters of both macro- and microeconomic systems, as well as their correlations and interactions in the determination of their states.

\section{Formulation of the problem}

The goal of the present article is the creation, based on statistical data of the development of an economic system, of an algorithm that will allow us to qualitatively determine their dynamic stability. This approach takes into account various non-linear effects in the dynamics of the system.

Unlike previous studies, we include both an analytical approach and a numerical approach.

\section{Results of the analysis.}

\subsection{Base equations}

We will analyze a one-dimensional model for an autonomous, conservative system. The dynamics of the system will be described by the following second-order differential equation (equation of motion):

$$
\frac{d^{2} x}{d t^{2}}=f(x)
$$

where $x$ is the deviation of the system from equilibrium, $f(x)$ is the "force" acting on the system, and $t$ is time. 


\section{Macrothink}

Research in Applied Economics

ISSN 1948-5433

2015, Vol. 7, No. 2

The right side of the equation (1) includes both linear and non-linear (in $\mathrm{x}$ ) terms. We remind the reader those terms linear in $\mathrm{x}$ lead to harmonic oscillations near equilibrium points. Nonlinear terms can lead to unstable states of the system, to an increase in the oscillation amplitude (the change of the parameters from their equilibrium values), and to the change of the frequencies themselves.

The qualitative description of the system by the method of the phase plane (the determination of how the potential function and the speed $d x / d t$ depend on $x$ ) requires us to know the explicit form of $\mathrm{f}(\mathrm{x})$. When economic systems depend on a large number of parameters, this becomes rather difficult to do. Numerical modeling also requires the explicit form of $f(x)$.

During the creation of the algorithm, the phase plane method will be used partially.

Recall that the equilibrium points are determined from the following equations:

$$
\frac{d x}{d t}=0 ; \quad \frac{d^{2} x}{d t^{2}}=f(x)=0,
$$

Meanwhile, the potential function $U(\mathrm{x})$ is

$$
f(x)=-\frac{\partial U}{\partial x}, \quad U(x)=-\int_{0}^{x} f(z) d z
$$

\subsection{The algorithm of the dynamic model}

In the algorithm of the dynamic model, we will use "Wolfram Mathematica". As a first step, we use the existing set of current statistical data to interpolate the dependency of the $(t)$, which characterizes the dynamics (deviation from equilibrium) of the economic systems. We interpolate it as a $(n-1)$-th order polynomial in $t$, where $n$ is the number of pairs of given data (base points). During cyclical changes in the state of a system, the periodic functions that describe the process can be interpolated by a power series. For the analysis, it is best to have a statistical set of no less than 8 points.

Next, we use the defined $x(t)$ and $f(t)$ to determine $f(x)$.

From the equation (2) we determine the equilibrium points, while from (3) we determine the potential function $U(x)$.

The potential function $U(x)$ can be determined in another way. The equation (1) can be brought to the following form:

$$
\frac{\partial x}{\partial t} * \frac{d^{2} x}{d t^{2}}=-\frac{\partial U}{\partial t}
$$

Using $x(t)$, we can obtain $U(t)$. Knowledge of $x(t)$ and $U(t)$ is sufficient to obtain $U(x)$.

By looking at the form of $U(x)$, we can easily determine "areas of stability" of the system. The minima of $U(x)$ determine stable equilibriums, while maxima determine unstable ones.

By analysing the sign of the second derivative of $U(x)$ 


$$
\frac{\partial f(x)}{\partial x}=-\frac{\partial^{2} U(x)}{\partial x^{2}}
$$

we can, both analytically and visually, determine the type of the extremum:

$$
\frac{\partial f(x)}{\partial x}=-\frac{\partial^{2} U(x)}{\partial x^{2}}<0-\min , \frac{\partial f(x)}{\partial x}=-\frac{\partial^{2} U(x)}{\partial x^{2}}>0-\max .
$$

\subsection{Application of the algorithm}

Let's give a concrete example. We have a set of eight pairs of observational data:

$$
(t, x)=(1,0.5),(2,0.55),(3,0.6),(4,0.62),(5,0.67),(6,0.78),(7,0.91),(8,1) .
$$

We can fit this data set by a seventh order polynomial in $t$. The results are shown in Fig. 1.

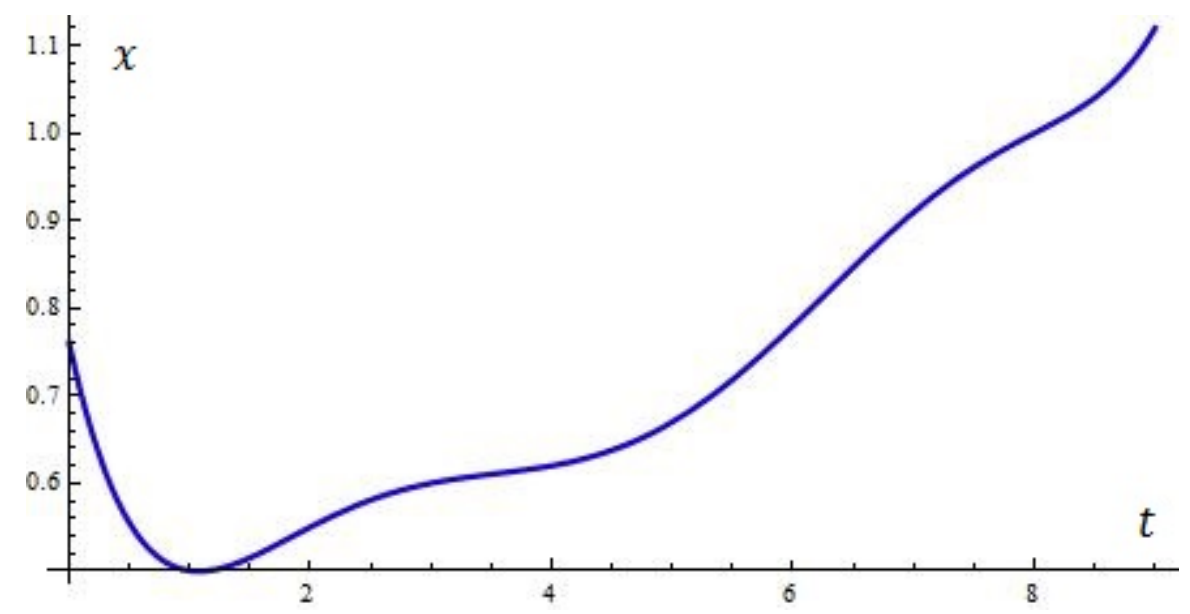

Figure 1. Interpolation of statistical data by a seventh order polynomial in $t$

Knowing $x(t)$ and $f=f(t)$ (through the second derivative of $x(t)$ ) allows us to determine $f(x)$ (Fig. 2).

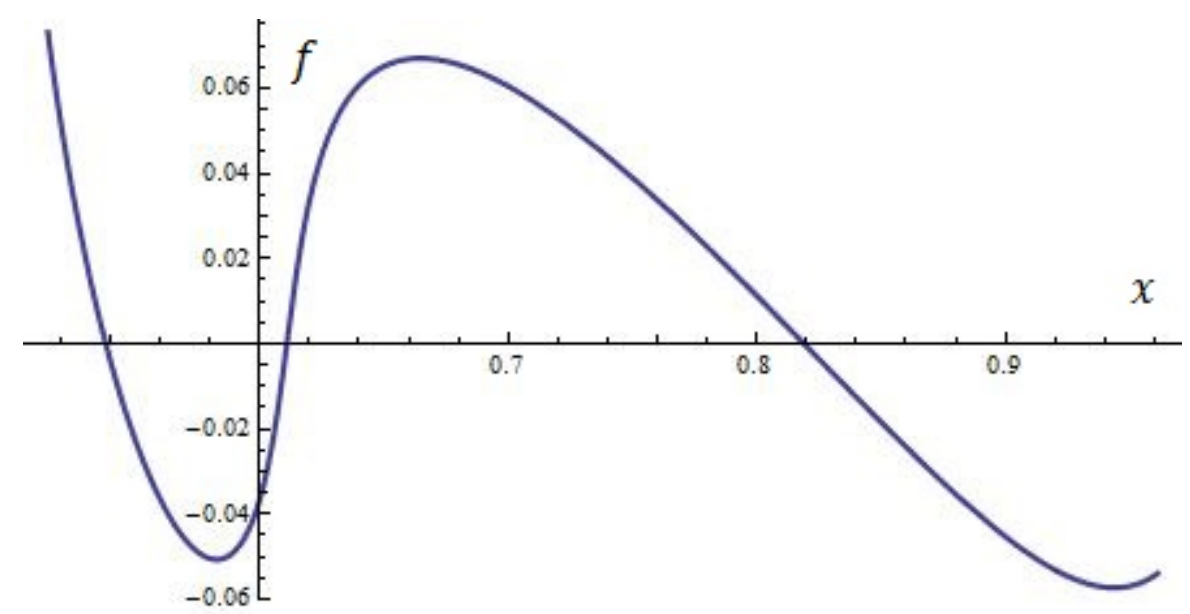

Figure 2. Dependency of the force $f$ that acts on the system on the system parameter $x$ From (2), we find the stationary points: $x_{1}=0.538, x_{2}=0.611, x_{3}=0.812, x_{4}=1$. 
By taking the derivative of $f(x)$, we can (graphically and analytically) determine the sign of this derivative in the stationary points.

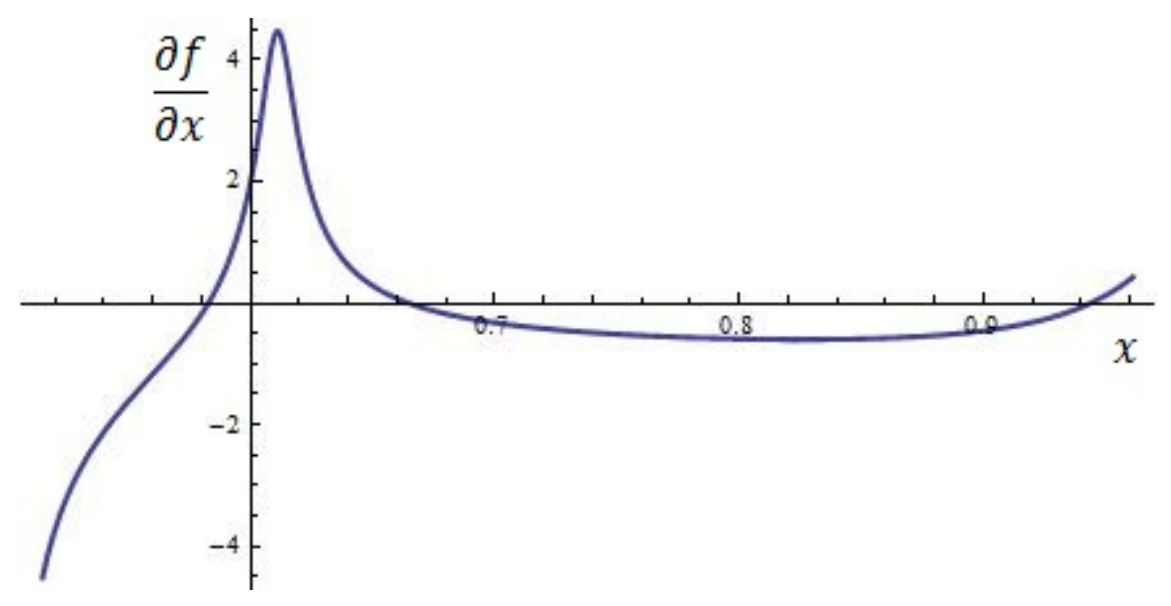

Figure 3. Dependency of the derivative of the force on the system parameter

From (5) and (6) we can find the extrema of the potential function of the system, and thus find its equilibrium states.

$$
\begin{aligned}
& \frac{\partial f\left(x_{1}\right)}{\partial x}=-\frac{\partial^{2} U\left(x_{1}\right)}{\partial x^{2}}<0-\min , \frac{\partial f\left(x_{2}\right)}{\partial x}=-\frac{\partial^{2} U\left(x_{2}\right)}{\partial x^{2}}>0-\max \\
& \frac{\partial f\left(x_{3}\right)}{\partial x}=-\frac{\partial^{2} U\left(x_{3}\right)}{\partial x^{2}}<0-\min , \frac{\partial f\left(x_{4}\right)}{\partial x}=-\frac{\partial^{2} U\left(x_{4}\right)}{\partial x^{2}}>0-\max
\end{aligned}
$$

The analytically and graphically determined extrema coincide. In the minima, the system is stable, while in the maxima, it is unstable.

The authors of the present article had, in an earlier paper, said that it would be fruitful to analyse how the stability of a system is determined by the form its potential function. The form of $U=U(x)$ can be determined by integrating $f(x)$. The results are shown on Fig. 4.

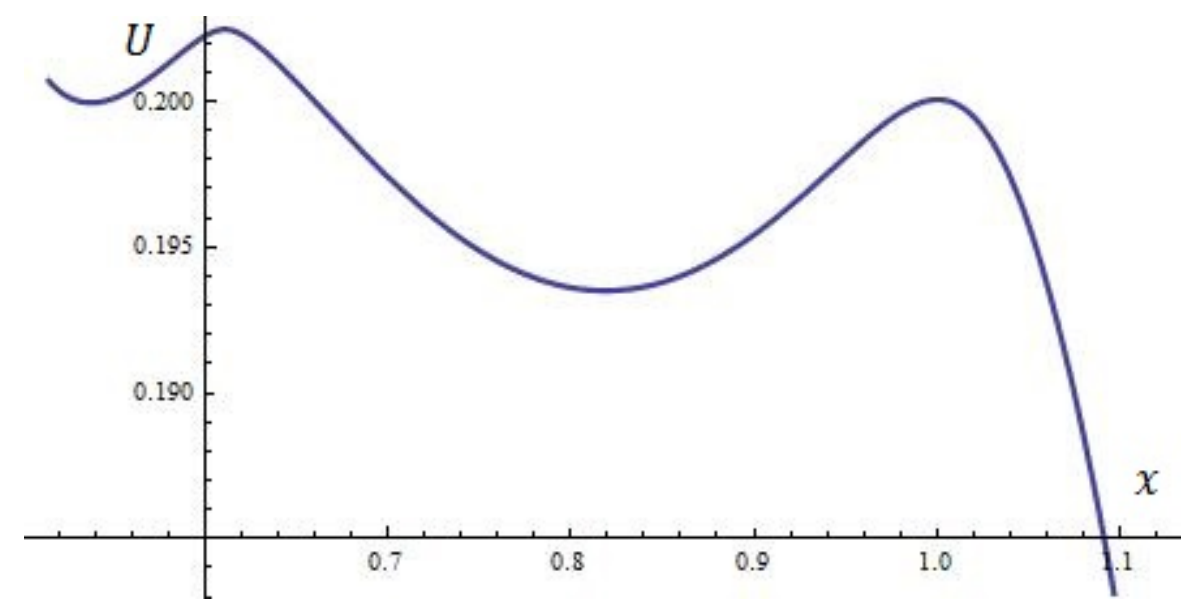

Figure 4. Dependency of the potential function of the system on the system parameter $x$

The extrema of $U=U(x)$ define equilibrium. 
It is worth noting that $U=U(x)$ is determined by the second method: we integrate (6) to get $U=U(t)$, and then use $x(t)$ to get $U=U(x)$.

The time-monitoring of the parameters that describe the dynamics of the system (the deviation from equilibrium) give statistical data that can be used with the proposed algorithm. Known methods of the approximate solution of equation (1) assume that the terms in the right side of $(f(x))$ are small. When this is not so, the numerical solution requires the explicit form of the force, as was mentioned before.

The reconstruction of the deviation of the system $(x)$ and its potential function $(U(x))$ with the help of a temporal parametric definition allows us to determine not only the values of the critical points, but also their extremal characteristics. Using statistical data, we determine the deviation and state of the system as a function of time. If an approach to a maximum point (unstable state) is noticed, immediate changes must be implemented to alter the tendencies of the system's development. By continuing to monitor the system's dynamical characteristics, we will always know which measures to implement in order to stabilize the system.

Let's analyze the example described in the article. With the help of statistical data (7), we determined the dependency of the deviation $x$ on time $t$ (see Fig. 1), as well as the dependency of the force $f=f(x)$ on the deviation (see Fig. 2). The critical points $x_{1}=0.538, x_{2}=0.611, x_{3}=0.812, x_{4}=1$ were determined analytically from equation (2), or simply by looking at where the graph of the force $f(x)$ intersected the $x$ axis. In addition to this, we calculated the derivatives of the force in the stationary (critical) points. It was found that $x_{1}$ and $x_{3}$ were minima, and that $x_{2}$ and $x_{4}$ were maxima. These same results are seen clearly on Fig. 3 - the graphical and analytical approaches yield the same result. The graph of the potential function $U(x)$ on Fig. 4 also shows the presence of the extrema defined above. The last value of the $x$ parameter in the dataset is equal to 1 . If the system then proceeds to shift to the side of $x<1$, then its oscillations will occur around a point of stable equilibrium. If the opposite happens $(x>1)$, the system will become unstable as it leaves the nearby points of equilibrium.

\section{Conclusion}

In the present article, an algorithm is constructed that allows us to determine the dynamical stability of economic systems by analysing a set of observational (statistical) data. The proposed approach gives the possibility of determining (analytically and graphically) the positions of the stable and unstable equilibria of the system. This approach does not require us to know the explicit form of the forces that act on the system. By comparing the current values of the parameter (that describes the state of the system) to the values of the extrema points, we can predict the dynamics of the system.

Let's apply the proposed method.

In the classical model of economics, assuming full employment, the oscillations of the employment market around equilibrium have a significant impact on the finance and 
commodities markets. In the Keynesian model, the level of employment $L$ depends on the demand for commodities $E$. Equilibrium in the commodities and finance markets defines the equilibrium state in the commodities market, which corresponds to a level of employment $L_{0}$ which is smaller than the level of full employment $L^{0}$, as defined by the classical model of economics.

In the simple case, when the value of demand for consumer and investor goods, as well as the speculative demand for currency, all depend linearly on their corresponding arguments (GDP for the first two, and interest rates for the latter) oscillations of the commodities and finance markets occur in the interest rate - GDP plane. The point of equilibrium of the commodities and finance markets is defined, predictably, as the point of intersection of the equilibrium lines of the commodities and finance markets. This point defines the equilibrium point of the employment market, which oscillates with its own frequency. The difference $L^{0}-L_{0}$ shows the value of the unemployment.

When monitoring the parameters of the markets, it is possible to determine their dynamics, whether they are stable or unstable. This is especially important during economic crises.

The proposed method can be used in conjunction with any economic structure, as long as supply and demand can be determined as functions of its parameters. For instance, let's look at the dependency of supply and demand of commodities on their cost. The point of intersection of the two dependencies defines the point of equilibrium of the system. Oscillations of the cost of the commodities occur around this point. The proposed algorithm allows us to determine the stability of a system with regards to external factors, which change the characteristics of the oscillations.

Future analysis should be focused on applying a similar algorithm to non-conservative economic systems.

\section{Acknowledgement}

The authors would like to thank Manifest Communications for their help during the research process.

\section{References}

Baba, I., \& Kenneth, O.-B. (2014). Analysis of the Goods Market and Money Market Equilibrium in a Developing Country. Modern Economy, 5(1), 105-111. http://dx.doi.org/10.4236/me.2014.51012

Brownlee, O.H. (1950). The Theory of Employment and Stabilization Policy. Journal of Political Economy, 58, 412-424. http://dx.doi.org/10.1086/256982

Brunner, K., \& Meltzer, A. (1964). Some Further Investigation of Demand and Supply Functions for Money. Journal of Finance, 19, 240-283. 
http://dx.doi.org/10.1111/j.1540-6261.1964.tb00767.x

Dankiv, V. V. (2012). Objective causes of economic oscillations and their effects on the labour market. Scientific vistnuk UU. Economics, 36, 25-28.

Ezekiel, M. (1938). The Cobweb Theorem. Quarterly Journal of Economics, 52, 255-280. http://dx.doi.org/10.2307/1881734

Forrester, J. W. (1973). World Dynamics (2nd ed.). Waltham, MA: Pegasus Communications.

Friedman, M. (1968). The role of monetary policy. American Economic Review, 58, 1-17.

Goodwin, R.M. (1991). Nonlinear Dynamics and Evolution. London: Macmillan.

Haghighi, H. K., Sameti, M., \& Isfahani, R. D. (2012). The Effect of Macroeconomic Instability on Economic Growth in Iran. Research in Applied Economics, 4(3), 39-61.

Kondratief, N. (1925). The static and the dynamic view of economics. The Quarterly Journal of Economics, 39, 575-583. http://dx.doi.org/10.2307/1883266

Kondratief, N. (2002). Big circles of state of business and foresight theory - selectas. Moscow: Economics.

Kostenko, E., Kuznichenko, V. M., \& Lapshyn, V. I. (2013). Influence of external periodic and non-linear factors upon stability of economic systems. The Problems of Economy, 2, 212-219.

Lorenz, H-W. (1993). Nonlinear Dynamical Economics and Chaotic Motion. Berlin: Verlag Springer. http://dx.doi.org/10.1007/978-3-642-78324-1

Macconel, C. P., \& Bru, S. L. (1993). Economics: principles, problems, politics. Moscow: Republic.

Mclean, R.D., \& Zhao, M. (2014). The business cycle, investor sentiment and castly external finance. The Journal of Finance, 69(3), 1377-1409.

Milovanov, V. (2001). Unstable socio-economic systems: synergy and self-organization. Moscow: Editorial URSS.

Mo, W.-S., Rahnamamoghadam, M., Summers, P., \& Valcarcel, V. (2014). Nonlinearities in the Economic Growth Rates of Taiwan and Hong Kong: A Bayesian Threshold Autoregression Approach. Research in Applied Economics, 6(2), 171-189. http://dx.doi.org/10.5296/rae.v6i2.5149

Petters, E. (1996). Chaos \& Order in the Capital Markets: A New View of Cycles, Prices and Market Volality (2nd ed.). New York. Toronto: John Wiley \& Sons, Inc. p. 288.

Pirtch, M. (2011). Causes of cyclical oscillations in Ukraine's economy. Vistnuk TNEU, 4, $18-25$.

Punzo, F. (2006). Cycle, Growth and Structural Change. London. New York: Taylor \& Francis e-Library. 


\section{Macrothink}

Research in Applied Economics

ISSN 1948-5433 2015, Vol. 7, No. 2

Rhee, H.J. (2010). Business cycle implification ofrate-based stabilization. International Journal of Economics, 2(2), 12-25.

Ruth, M., \& Hannon, B. (1997). Modeling Dynamic Economic Systems. New York: Springer. http://dx.doi.org/10.1007/978-1-4612-2268-2

Smirnov, A. D. (2000). Lectures on macroeconomic modeling. Moscow: GU VSE.

Tobin, J. (1978). Monetary policies and the economy: the transmission mechanism. Southern Economic Journal, 44, 421-431. http://dx.doi.org/10.2307/1057201

Turnovsky, S. J. (2000). Methods of Macroeconomic Dynamics (2nd ed.). Boston: MIT Press.

Verhulst, F. (1997). Nonlinear Differential Equations and Dynamical Systems. Berlin: Verlag Springer.

Vnukova, N., \& Voronin, A. (2010). Modifications of the M.Kaletsky model, which describes the dynamics of capital. Economic of Ukraine, 1, 4-10.

\section{Copyright Disclaimer}

Copyright for this article is retained by the author(s), with first publication rights granted to the journal.

This is an open-access article distributed under the terms and conditions of the Creative Commons Attribution license (http://creativecommons.org/licenses/by/3.0/). 